\title{
ANÁLISIS DE LA INTENCIÓN EMPRENDEDORA EN ESTUDIANTES UNIVERSITARIOS: VALIDACIÓN DE LA TEORÍA DE ACCIÓN PLANIFICADA
}

\section{ANALYSIS OF ENTREPRENEURIAL INTENTION IN UNIVERSITY STUDENTS: VALIDATION OF THE THEORY OF PLANNED ACTION}

Edison Ignacio Espinoza Alcívar, Mgs. Magíster en dirección y gestión de empresas de servicio (Ecuador). Docente de la Facultad de Ciencias Administrativas de la Universidad de Guayaquil, Ecuador. edisonespinozaa@ug.edu.ec

\section{Juan Antonio Jimber Del Río, Ph.D.} Doctor en Ciencias Sociales y Jurídicas de la Universidad de Córdoba (España). Docente Ayudante Doctor de la Facultad de Derecho y Ciencias Económicas y Empresariales de la Universidad de Córdoba, España.

jijimber@uco.es

José Carlos Casas del Rosal, Ph.D.

Doctor en Ciencias Sociales y Jurídicas de la Universidad de Córdoba (España). Profesor asociado del Departamento de Estadística, Econometría e Investigación Operativa de la Universidad de Córdoba, España.

jcasas@uco.es

Nuria Ceular Villamandos, Ph.D.

Doctora en Ciencias Económicas y Empresariales de la Universidad de Córdoba (España). Directora del Departamento de Estadística, Organización de Empresas y Estadística Aplicada de la Universidad de Córdoba, España.

\section{ARTÍ́CULO DE INVESTIGACIÓN}

Recibido: 10 de septiembre de 2018.

Aceptado: 5 de noviembre de 2018. 


\section{RESUMEN}

El presente trabajo tiene como propósito medir la intención emprendedora en estudiantes universitarios tomando en consideración la Teoría de la Conducta Percibida (TCP) desarrollada por Ajzen (1991), el instrumento utilizado es un Cuestionario de Intención Emprendedora (CIE) desarrollado por Krueger (2000) y aprobado en España por Rueda (2011), esta encuesta fue enviada a 1430 correos de estudiantes de diferentes universidades tanto pública como privadas de la Zona administrativa 8 que comprenden los cantones de Guayaquil, Durán y Samborondón en Ecuador, en la que se obtuvo una tasa de respuesta del $21 \%$, se realizó un análisis descriptivo correlacional, donde se obtuvo como resultado que existe un alto índice de intencionalidad en el grupo estudiado y que es explicado fuertemente por la actitud hacia la conducta y por la autoeficacia percibida, lo que comprueba la fiabilidad y validez del instrumento utilizado.

Palabras Clave: Emprendimiento, Intencionalidad, Comportamiento planificado.

\section{ABSTRACT}

The purpose of this paper is to measure the entrepreneurial intention in university students to take into account the theory of perceived behavior (TCP) developed by Ajzen (1991), the instrument is a Questionnaire of Intent Entrepreneurship (CIE) developed by Krueger (2000) and approved in Spain by Rueda (2011), this survey was delivered to 1430 emails from students from different universities, both public and private, from Administrative Zone 8, which delivers the cantons of Guayaquil, Durán and Samborondón in Ecuador, in which there was a response rate of $21 \%$, a correlational descriptive analysis was carried out, which resulted in a high index of intentionality in the group studied and that is strongly explained by the attitude toward behavior and by the perceived self-efficacy, which confirms the reliability and the validity of the instrument used.

Keywords: Entrepreneurship, Intentionality, Planned behavior.

\section{INTRODUCCIÓN}

Un estudio realizado por el Global Entrepreneurship Monitor (GEM), reveló que en Ecuador se evidencia un alto índice de la Actividad Emprendedora Temprana con respecto a otros países de Latinoamérica y el Caribe involucrados en este estudio (ESPAE, 2018) este índice, agrupa a la población entre los 18 a 64 años que estén iniciando un emprendimiento, o que ya lo tengan en marcha pero que no hayan superado aún los tres años y medio de funcionamiento.

Este mismo estudio, también destaca que entre los factores que promueven el emprendimiento son: la educación profesional y continua, la infraestructura comercial, las normas sociales y 
culturales, mientras que los factores más relevantes que limitan el emprendimiento, son: el financiamiento, las políticas gubernamentales y la capacidad para emprender. Por lo que, se entiende que todo emprendimiento requiere un proceso minucioso compuesto por diferentes fases que deben ser planificadas (Schnarch, 2014), es justamente a la primera fase del proceso emprendimiento donde se han realizado numerosos estudios (Moriano, Gorgievski, Laguna, Stephan \& Zarafshani, 2012; Liñán, Nabi \& Krueger, 2013; Krueger, 2017) en ellos se ha evaluado la intención hacia el emprendimiento y se ha confirmado que la intencionalidad es el mejor predictor para una conducta emprendedora.

La Teoría del Comportamiento Planificado desarrollado por Ajzen (1991), es el modelo más utilizado para estudiar la intención emprendedora y también es utilizada en numerosos estudios para predecir la intención conductual en diferentes áreas. Como instrumento estandarizado para evaluar la intencionalidad, se utilizará el Cuestionario de Intención Emprendedora (Rueda et al., 2011) y que se utilizará en esta investigación para mantener un criterio uniforme y comparable de los resultados que se obtengan.

El objetivo de esta investigación, es analizar los factores que influyen en la intención emprendedora de los estudiantes universitarios mediante la validación de un instrumento que abarca la Teoría de Comportamiento Planificado.

\section{REVISIÓN TEÓRICA}

Existen numerosos autores que relacionan de forma directa el emprendimiento y la competitividad (Amorós \& Cristi, 2008; Galindo \& Méndez, 2011), convirtiendo al emprendimiento en uno de los factores que aporta al desarrollo económico de las naciones (Acs, Szerb \& Lloyd, 2017). En países Latinoamericanos y el Caribe existe una relación positiva entre la actividad emprendedora y el crecimiento económico, tomando en consideración que el tipo de emprendimiento más notorio es el impulsado por la necesidad (Amorós, Borraz \& Veiga, 2016). Aunque, en estas regiones existe una cultura muy fuerte hacia la dependencia laboral, obligada por la inseguridad económica y las políticas paternalistas de sus gobiernos (Durán \& Arias, 2015).

Por otro lado, también se puede mencionar la relación directa que existe entre el nivel de estudios y la intención de iniciar un emprendimiento (Heuer \& Kolvereid, 2014), por lo que se espera que a mayor nivel de estudios o preparación de los individuos exista una intención positiva de iniciar un negocio. 
El emprendimiento es la capacidad y habilidad para diseñar, organizar y gerenciar una empresa cuyo resultado sean bienes o servicios, donde el emprendedor se enfrenta a los riesgos necesarios con el fin de obtener un beneficio económico (Castro, García \& Adame, 2015). El ejemplo más innegable de emprendimiento es el nacimiento de nuevos negocios. En economía, el emprendimiento combinado con la tierra, la mano de obra, los recursos naturales y el capital puede generar ganancias. El espíritu emprendedor se caracteriza por la innovación y la asunción de riesgos, y es una parte esencial de la capacidad de una nación para tener éxito en un mercado global en constante cambio y cada vez más competitivo (Moreno, 2015).

El complejo proceso de desarrollo de un emprendimiento tiene sus inicios en la intención de llevarla a cabo (Valencia Arias, Montoya \& Montoya, 2014). La complejidad del proceso de creación de un negocio depende del tamaño del emprendimiento (García, 2015).

La intencionalidad se puede definir como el estado consciente de la mente que precede a la acción. Esta intención establece la forma del comportamiento de los individuos afectando individualmente sus decisiones (Arango, Clavijo, Duque \& Puerta, 2014).

Existen algunos modelos para evaluar y explicar la intención emprendedora. El primero está basado en el evento emprendedor, el cual sugiere que, para que un individuo inicie una actividad empresarial, debe considerar que le es factible realzarla y alcanzar el éxito, además, debe tener una inclinación a no desaprovechar las oportunidades (Shapero \& Sokol, 1982). Otro de los modelos, es el modelo bidimensional utilizado en estudiantes universitarios en Venezuela, la escala contenía factores como el posicionamiento en el mercado, y se refiere a que cada individuo tiene la capacidad de entablar relaciones con potenciales inversionistas. Otro de los factores de este modelo es el desarrollo de productos, que es la creencia sobre la capacidad de administrar todos los temas referentes al negocio. Liderazgo de recursos claves, que es el sentimiento del individuo para dirigir equipos de trabajo y lograr sinergia y empoderamiento en la organización indistintamente del tipo de negocio (Salvador, 2008).

Otro modelo plantea la evaluación de la intención emprendedora mediante un conjunto de factores cognitivos como la autoeficacia, la actitud hacia emprender y el estilo emocional, y variables socio personales como vivir con los padres, experiencia laboral, tener referentes familiares de emprendimiento (Durán \& Arias, 2015).

En algunas investigaciones sobre intención emprendedora solo se utilizan escalas simples sin validación y métricas no estandarizadas (Sánchez, Boada, Prizmic \& Hernández, 2014). Sin embargo, estos trabajos no han sido tan acreditados como los que emplean la Teoría de Comportamiento Planificado (TCP), esta teoría fue desarrollada por Ajzen (1991) y se fundamenta 
en explicar el comportamiento intencionado en base a factores personales y sociales, esta teoría de índole sociocognitiva se ha aplicado con éxito en distintas áreas. EI TCP establece un marco teórico coherente y aplicable, el cual permite predecir la intención emprendedora tomando en cuenta no solo los factores personales sino también los sociales (Krueger, Reilly, \& Carsrud, 2000). La TCP está conformada por tres componentes que definen la intención hacia la conducta: actitud hacia la conducta, norma subjetiva, y el control conductual percibido. Estos componentes predicen la intención emprendedora de una forma más directa.

El componente de actitud hacia la conducta está condicionado por el conjunto total de las creencias conductuales que vinculan el comportamiento con diversos resultados (Icek Ajzen, Heilbroner, Fishbein \& Thurow, 1980), es decir, la actitud es una emoción o estado mental de alerta que pone al individuo en una situación positiva o negativa y que ejerce influencia sobre su comportamiento futuro hacia otras personas, objetos o situaciones en base a la experiencia o el aprendizaje. Por lo tanto, dos sujetos pueden tener la misma convicción de que emprender puede significar la confrontación de una serie de retos, pero uno de ellos puede percibir estos retos de una manera positiva mientras que el otro puede verlo de forma negativa (Rueda et al., 2011).

Por su parte la norma subjetiva, es definida por Ajzen (1991), como la percepción que tiene cada individuo a las presiones sociales para involucrarse o no en un comportamiento de tipo empresarial, entre estas normas se pueden mencionar las influencias filiales sobre la esperanza que ellos tienen del tipo de profesión o emprendimiento que se pueda llegar a realizar (TorresHernández, Barreto \& Vásquez, 2015). La norma subjetiva refleja dos sub componentes: las creencias normativas, que es la norma que establecen los referentes importantes de cómo debe comportarse el individuo y la motivación de cumplimiento, que es la voluntad de comportarse de acuerdo a lo que esperan los referentes (Icek Ajzen et al., 1980). Este componente depende del entorno social en que se desenvuelvan los sujetos de estudio provocando que su comportamiento aumente o disminuya los indicadores de la intencionalidad en el emprendimiento (Lortie \& Castogiovanni, 2015; Rodriguez, 2015).

El tercer componente es el control conductual percibido, se refiere básicamente a la percepción subjetiva que tiene el individuo de su capacidad para realizar un comportamiento (Icek Ajzen, 1991). En estudios sobre intención de emprendimiento la autoeficacia sustituye al control conductual percibido ya que ambos conceptos se refieren a la capacidad percibida para expresar una conducta, por ejemplo, iniciar un negocio. Para Bandura (2001), la autoeficacia es la creencia de los individuos sobre su capacidad para cumplir con éxito ciertas actividades, para esto la elaboración de los ítems del instrumento de recolección de datos deben ser específicos y no hechos de forma general. Por su parte Armitage y Conner (2010), concluyen en su estudio que 


\section{Revista Científica ECOCIENCIA}

la autoeficacia se define de forma más clara y presenta una mayor correlación con las intenciones de emprender que el control conductual percibido.

\section{MATERIALES Y MÉTODOS}

Por lo anteriormente mencionado, se puede establecer que los tres componentes de la TCP, actitud hacia la conducta, las normas subjetivas y el control conductual percibido, que es medido mediante la auto eficacia, agrupan la mayor cantidad de información suficiente para predecir la intención hacia el comportamiento de los individuos. Por tanto, se espera como resultado una relación positiva en todos los componentes con la intención emprendedora, las hipótesis de investigación se formulan de la siguiente forma:

Hipótesis 1: la actitud hacia la conducta emprendedora se relaciona de manera positiva con la intención hacia el emprendimiento.

Hipótesis 2: la norma subjetiva tiene una relación positiva con la intención hacia el emprendimiento.

Hipótesis 3: la autoeficacia se relaciona positivamente con la intención hacia el emprendimiento.

H 1: Actitud hacia la conducta

H 2: Norma subjetiva

H 3: Autoeficacia
Intención hacia el emprendimiento

Figura 1. Interpretación gráfica de las hipótesis (Tomado de: Rueda et al., 2011)

Se utilizó una cuestionario validado en el trabajo de Laguía (2017) quien realizó una evaluación de la TCP en estudiantes universitarios en Colombia, este cuestionario fue desarrollado para un estudio realizado en España con el fin de validar la Teoría de Comportamiento Planificado (Rueda et al., 2011), además, fue adaptado al entorno y condiciones de las universidades, con el diseño del cuestionario también se logró estandarizar la obtención de los datos, de tal manera, que pueda haber un análisis comparativo con otros estudios sobre intención hacia e comportamiento. 
El cuestionario se llevó a cabo de manera online mediante la herramienta de Google Forms (Google Forms, 2018), en la que se enviaron 1430 correos electrónicos a estudiantes universitarios que están cursando los últimos años de estudios de sus carreras. La cumplimentación se realizó de manera voluntaria y anónima y la tasa de respuesta fue de $21 \%$. Formalizando una muestra de 303 estudiantes de diferentes universidades entre públicas y privadas. Para la validación del cuestionario y la elaboración de la parte descriptiva y correlacional de los datos, se utilizó el paquete estadístico Statistical Package for Social Science SPSS v.22.

El cuestionario está dividido en cuatro partes compuesto de 35 items: la intención emprendedora (5 items), la actitud hacia la conducta emprendedora (12 items), las normas subjetivas (6 items) y la autoeficacia (12 items), todos los ítems son elaborados de forma enunciativa y la valoración de cada uno de ellos se lleva a cabo a través de una escala Likert de 10 niveles, desde el valor 1 que se corresponde con una posición de total desacuerdo y el 10 con una totalmente de acuerdo. Siguiendo los trabajos anteriores en los que se utilizó el Cuestionario de Intención Emprendedora CIE, la intencionalidad se midió utilizando una escala de cuatro componentes mediante la cual un sujeto evalúa la probabilidad elegir una ocupación profesional, por ejemplo: ser abogado, ingeniero o emprendedor, expresando esta intencionalidad con los valores más altos. La fiabilidad de esta escala, como se observa en la tabla 1, alcanzó un índice del Alfa de Cronbach de 0.98, lo que indica que es muy satisfactoria.

Las actitudes hacia la conducta emprendedora se midieron con un conjunto de seis afirmaciones sobre la expectativa de iniciar un negocio propio y otro conjunto de seis situaciones sobre su deseabilidad en cuanto a esta acción, luego los resultados se ponderaron y se agregaron los resultados para para obtener el puntaje de la escala, esto se lo realizó mediante la herramienta para calcular variable del paquete estadístico SPSS (Kautonen, van Gelderen \& Tornikoski, 2013). El Alfa de Cronbach en este grupo de preguntas alcanza un índice de 0.98 por lo que se considera como satisfactoria ya que el valor mínimo debe ser de 0.7 (Nunnally \& Bernstein, 1998).

Tabla 1. Fiabilidad de las escalas.

Alfa de Cronbach

\begin{tabular}{ll}
\hline Intención emprendedora & 0,98
\end{tabular}

$\begin{array}{lr}\text { Actitud hacia la conducta } & 0,98\end{array}$

$\begin{array}{ll}\text { Norma subjetiva } & 0,90\end{array}$

$\begin{array}{ll}\text { Autoeficacia } & 0,97\end{array}$ 
Fuente: Elaboración propia.

Las normas subjetivas se midieron con un grupo de tres afirmaciones sobre como valorarían los referentes significativos (como familia, amigos o conocidos) la decisión del individuo a iniciar un emprendimiento y otras tres afirmaciones sobre cómo valora la opinión esos referentes, estos dos grupos se promediaron para obtener el puntaje de la escala. El índice de fiabilidad en este conjunto de preguntas alcanzó 0.90 . La norma subjetiva está determinada por el conjunto total de creencias reguladas accesibles con respecto a las expectativas de los referentes importantes. Específicamente, la fuerza de cada creencia normativa (n) se pondera por la motivación para cumplir $(m)$ con el referente en cuestión, y los productos se agregan.

La autoeficacia empresarial fue medida mediante un grupo de seis afirmaciones las cuales se promediaron para obtener un solo valor de la escala, el Alfa de Cronbach alcanzó un índice de 0.97, lo cual se considera como aceptable. Como se mencionó anteriormente, esta escala medirá la percepción que tiene cada individuo en realizar tareas como captar inversionistas, gestionar el recurso humano, administrar aspectos concernientes al giro del negocio.

Además, se tomaron en cuenta otras variables de índole social y demográficas como: el género de los encuestados de los cuales el $63 \%$ son mujeres y el $37 \%$ hombres, la experiencia como emprendedor en la que se obtuvo como resultado que el $39.6 \%$ han tenido experiencia iniciando un negocio, sin embargo, de este grupo el $84.5 \%$ lo ha tenido por menos de un año, en cuanto al nivel social al que pertenece el $54.8 \%$ considera que es de un nivel medio, el $30.7 \%$ de un nivel medio bajo y el $2 \%$ de nivel bajo, el resto corresponde a niveles medio alto y alto. El nivel de estudio alcanzado por sus padres demuestra un valor entre $60 \%$ y $70 \%$ en los niveles de primaria y bachillerato para ambos casos.

\section{ANÁLISIS DE RESULTADOS}

Como resultado se puede mencionar que en cuanto al análisis correlacional y descriptivo las variables estudiadas presentan una dependencia positiva y significativa. En la tabla 2, se observa que la media de la intención emprendedora fue de 8 con una desviación estándar de 1.89, esto da a entender que la mayoría de las personas entrevistadas tiene una inclinación a emprender en un futuro inmediato debido a la calificación cercana a total acuerdo. 
Tabla 2. Estadísticos descriptivos.

\begin{tabular}{lcc}
\hline & Media & Desviación estándar \\
\hline Intención emprendedora & 8,0000 & 1,88827 \\
Actitud hacia la conducta & 8,0747 & 2,39086 \\
Norma subjetiva & 6,9125 & 2,50232 \\
Autoeficacia & 8,4560 & 1,84620 \\
\hline
\end{tabular}

Fuente: Elaboración propia.

La variable autoeficacia obtuvo una media de 8.5 siendo la más alta con una desviación estándar de 1.84, esto supone que la mayoría de los encuestados se siente en la capacidad de iniciar un negocio. Así mismo, como se mencionó en la literatura, la variable de norma subjetiva resultó con una media de 6.9 siendo la más baja, se puede entender que los factores en los que influyen los referentes sociales no tienen un fuerte dominio sobre la intención de emprender, como se mencionó en la revisión literaria, esto se debe principalmente al entorno en el cual se desenvuelven los individuos objetos del estudio.

En cuanto al análisis de las correlaciones entre las variables latentes, se puede observar en la tabla 3, que las que tienen mayor relación con la intención emprendedora son la Actitud hacia la conducta y la autoeficacia, se puede evidenciar que la norma subjetiva presenta una baja correlación en comparación con los otros componentes de la TCP. Sin embargo, también se puede apreciar que entre los componentes Actitud hacia la conducta y la autoeficacia existe un alto grado de correlación 0.818 ya que este valor es el más próximo a uno, es de suponer que si un individuo tiene una fuerte creencia que posee las capacidades necesarias para iniciar un emprendimiento, tendrá una fuerte inclinación hacia un comportamiento emprendedor y, por consiguiente, su intencionalidad de emprender será alta.

Tabla 3. Correlaciones

$\begin{array}{cccc}\text { Intención } & \begin{array}{c}\text { Actitud } \\ \text { hacia la } \\ \text { emprendedora }\end{array} & \begin{array}{c}\text { Norma } \\ \text { subjetiva }\end{array} & \text { Autoeficacia } \\ & \text { conducta } & & \end{array}$




\begin{tabular}{|c|c|c|c|c|c|}
\hline $\begin{array}{l}\text { Actitud hacia } \\
\text { la conducta }\end{array}$ & $\begin{array}{l}\text { Coeficiente de } \\
\text { Pearson }\end{array}$ &, $723^{\star *}$ & 1 &, $597^{\star \star}$ &, $818^{* \star}$ \\
\hline & Sig. (bilateral) & ,000 & & ,000 & ,000 \\
\hline $\begin{array}{l}\text { Norma } \\
\text { subjetiva }\end{array}$ & $\begin{array}{l}\text { Coeficiente de } \\
\text { Pearson }\end{array}$ &, $618^{* *}$ &, $597^{\star *}$ & 1 &, $597^{\star \star}$ \\
\hline & Sig. (bilateral) & ,000 &, 000 & & ,000 \\
\hline Autoeficacia & $\begin{array}{l}\text { Coeficiente de } \\
\text { Pearson }\end{array}$ &, $722^{* *}$ &, $818^{* *}$ &, $597^{* *}$ & 1 \\
\hline & Sig. (bilateral) & ,000 &, 000 & ,000 & \\
\hline
\end{tabular}

**. La correlación es significativa en el nivel 0,01 (2 colas).

Fuente: Elaboración propia.

La validez convergente representa la varianza común entre los indicadores y su construcción, y significa que un conjunto de indicadores está midiendo el mismo constructo subyacente (Henseler, Ringle \& Sinkovics, 2009), Schumacker (2017) recomienda usar como criterio la varianza promedio extraída (AVE). Cuanto mayor sea el valor de AVE, más distintivos son los indicadores del constructo en la que se cargan las variables latentes.

En general, su valor debe ser superior a 0.50 (Schumacker, 2017). Como se muestra en la Tabla 4 , el AVE para cada constructo fue satisfactorio, esta se obtuvo a partir de las cargas factoriales de cada ítem. Se puede observar que estos valores son mayores a 0.6 (Levy \& Mallou, 2006) por lo que se considera aceptable. También se obtuvo el coeficiente de fiabilidad de las medidas (Hair, Black, Anderson \& Babin, 2018), con el fin de evaluar la escala ya que es más conveniente que el Alfa de Cronbach, debido a que la fiabilidad de este modelo no depende del número de particularidades que estén asociados a cada concepto.

Tabla 4. Carga factorial, AVE y Coeficiente de fiabilidad.

\begin{tabular}{lcccc}
\hline \multicolumn{1}{c}{ Variable Latente } & Item & $\lambda$ & AVE & Cf \\
\hline Actitud hacia emprender & AE1 &, 76 &, 571 &, 889 \\
& AE2 &, 75 & & \\
AE3 &, 77 & & \\
AE4 &, 74 & & \\
& AE5 &, 78 & & \\
Norma Subjetiva & AE6 &, 73 & & \\
\hline
\end{tabular}




\begin{tabular}{lllll} 
& NS2 &, 89 & & \\
& NS3 &, 85 & & \\
\hline Autoeficacia & EF1 &, 81 &, 665 &, 922 \\
& EF2 &, 84 & & \\
EF3 &, 82 & & \\
EF4 &, 82 & & \\
EF5 &, 83 & & \\
\hline Intención & EF6 &, 79 & & \\
emprendedora & IE1 &, 78 &, 678 & \\
& IE2 &, 82 & & \\
IE3 & & & \\
IE4 &, 84 & & \\
IE5 &, 85 & & \\
\hline
\end{tabular}

Fuente: Elaboración propia.

Finalmente, se realizó un modelo de regresión para determinar el coeficiente de determinación, el cual se puede observar en la tabla 5 corresponde a 0.780 que es aceptable, y el R cuadrado de 0.680 indicando que el $60.8 \%$ de la variabilidad de la intención hacia la intención emprendedora se predice en base a las influencias de los componentes de la TCP.

Tabla 5. Resumen del modelo de regresión

\begin{tabular}{lrrrrr} 
Modelo & $\mathrm{R}$ & $\mathrm{R}$ cuadrado & $\begin{array}{c}\text { R cuadrado } \\
\text { ajustado }\end{array}$ & $\begin{array}{c}\text { Error estándar } \\
\text { de la } \\
\text { estimación }\end{array}$ & $\begin{array}{c}\text { Durbin- } \\
\text { Watson }\end{array}$ \\
\hline 1 &, $780^{\mathrm{a}}$ &, 608 &, 604 & 1,18753 & 2,086 \\
\hline
\end{tabular}

a. Predictores: (Constante), Autoeficacia, Norma subjetiva, Actitud hacia la conducta

b. Variable dependiente: Intención emprendedora

Fuente: Elaboración propia.

Los coeficientes beta o coeficientes estandarizados permiten determinar cuál es la variable explicativa que tiene mayor peso. Se puede observar en la figura 2, que con respecto a la relación entre las variables de la TCP, la actitud hacia la conducta emprendedora presenta una mayor influencia sobre la intención emprendedora obteniéndose resultados como $\beta=0.322, p<0.05$; seguida de la variable autoeficacia que obtuvo como resultado $\beta=0.318, p<0.05$; en contraste 


\section{Revista Científica ECOCIENCIA}

las normas subjetivas obtuvieron menor valor en la evaluación $\beta=0.235, p<0.05$ confirmando así lo mencionado por otros autores sobre la poca la influencia de esta variable sobre la intención emprendedora.

En forma general, los componentes de TCP pudieron explicar un alto porcentaje de la varianza de la intención emprendedora $\mathrm{R}^{2}=0.608$ que es una indicación significativa de la validez predictiva del cuestionario TCP.

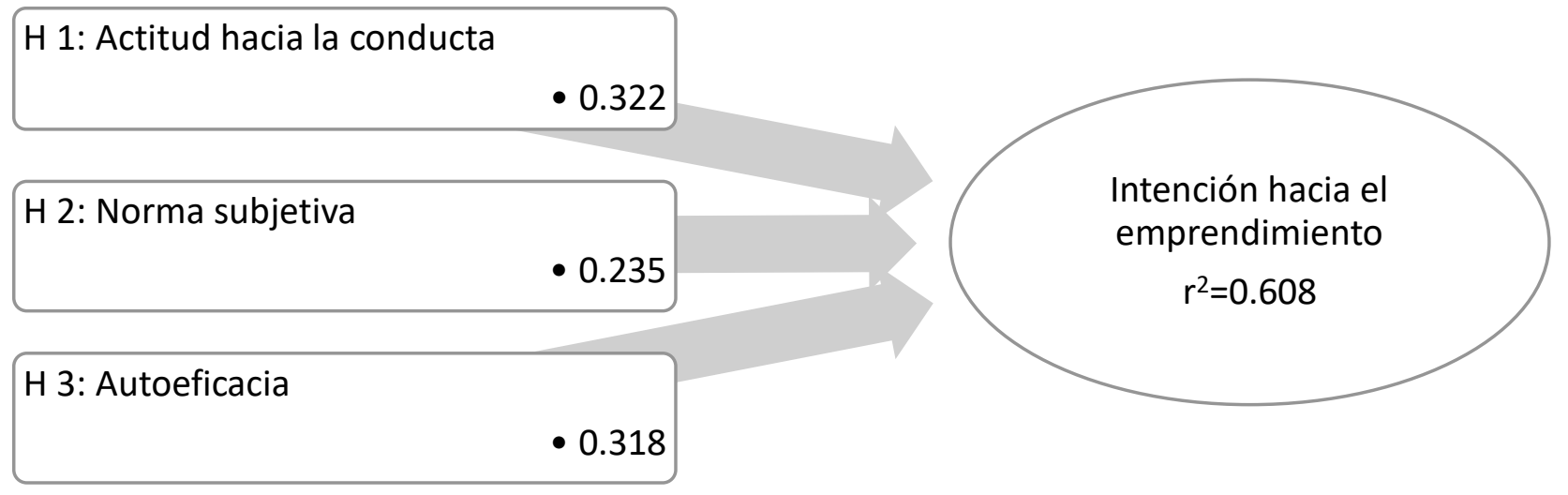

Figura 2. Resultados de las hipótesis (información obtenida del análisis estadístico)

\section{CONCLUSIONES}

Se puede mencionar, por tanto, que los tres componentes de la Teoría del Comportamiento Planificado permiten predecir la intención de emprendimiento en los individuos. En el caso de la actitud hacia la conducta, los estudiantes universitarios demuestran tener un fuerte conjunto de creencias necesarias para afrontar los retos que el inicio de un negocio representa.

En cuanto a la autoeficacia, los resultados en los estudiantes también demostraron una alta influencia de la percepción de la capacidad que tienen para iniciar un emprendimiento. Este resultado no tiene una diferencia significativa en los estudiantes de las universidades públicas como privadas. Los datos sugieren, que el tener conocimientos relacionados con su futura profesión, habilita a los estudiantes a tener confianza en su desenvolvimiento para iniciar un emprendimiento. En trabajos similares realizados con universitarios, también se encuentra estos altos valores en el factor de autoeficacia. 
La norma subjetiva, que mide la influencia social percibida del entorno donde se ha desarrollado el emprendedor y la importancia que le da los referentes externos, no tiene una fuerte influencia mayor comparada con las demás variables, sin embrago, cuando se analiza los resultados de las universidades privadas de manera individual, los valores de este factor, son más altos que en el de las universidades públicas, esto supondría, que los estudiantes universitarios de instituciones privadas tienen mayor influencia social en el aspecto emprendedor debido a la experiencia de su entorno.

Se evidencia con los resultados, que el cuestionario CIE utilizado es una herramienta fiable para realizar estudios sobre la intención de emprendimiento en varias áreas por lo que se puede utilizar para hacer estudios comparativos de las intenciones de emprendimiento entre diferentes grupos de individuos.

Del mismo modo, la posibilidad de que algunos efectos de interacción puedan estar presentes entre algunos de los antecedentes motivacionales de la intención, merece una mayor investigación (Fitzsimmons \& Douglas, 2011). En este sentido, podría ser útil el análisis mediante ecuaciones estructurales, en diferentes muestras, para comparar la estructura, tanto del modelo básico de TCP como el modelo modificado que se desarrolle. El cuestionario CIE facilita estas comparaciones, permitiendo tener la certeza que los resultados serán confiables y comparables. El uso de estudiantes como muestra de estudio, aunque es viable, está sujeto a críticas (Robinson, Stimpson, Huefner \& Hunt, 1991). En este trabajo, se discute que es un grupo singular de la población con poca experiencia real y responsabilidades limitadas. Por lo tanto, puede no ser representativo de la población adulta en general. Sin embargo, la tendencia de los estudiantes universitarios es de ingresar al mercado laboral justo después de la graduación o inclusive antes, tomando responsabilidades en el trabajo relativamente más temprano que otros grupos menos instruidos. Además, las personas con educación universitaria en el grupo de edad de 25 a 34 años están especialmente inclinadas hacia el emprendimiento (Aliaga \& Schalk, 2018; Soria, Zuniga \& Ruiz, 2016). En Ecuador, más del 50\% del grupo de emprendedores nacientes contempla el rango de edad entre 18 y 34 años (ESPAE, 2018), por lo tanto, la muestra utilizada es representativa de un segmento de población grande y significativo.

Como limitante del estudio se puede mencionar la característica transversal de los datos obtenidos, un estudio de forma longitudinal podría mostrar si aquellos estudiantes que presentan mayor intencionalidad de emprendimiento lograron iniciar un negocio. 


\section{REFERENCIAS BIBLIOGRÁFICAS}

Acs, Z. J., Szerb, L., \& Lloyd, A. (2017). The Global Entrepreneurship and Development Index. SpringerBriefs in Economics. Springer, Cham. https://doi.org/https://doi.org/10.1007/978-3319-65903-9_3

Ajzen, I. (1991). The Theory of Planned Behavior. Organizational Behavior and Human Decision Processes, 50(2), 179-211. https://doi.org/https://doi.org/10.1016/0749-5978(91)90020-T

Ajzen, I., Heilbroner, R. L., Fishbein, M., \& Thurow, L. (1980). Understanding attitudes and predicting social behavior. Prentice Hall PTR.

Aliaga, C., \& Schalk, A. (2018). E2: Empleabilidad temprana y emprendimiento. Dos grandes desafíos en la formación superior en Chile. Calidad En La Educación, 33, 319-337. https://doi.org/https://doi.org/10.31619/caledu.n33.145

Amorós, J. E., Borraz, F., \& Veiga, L. (2016). Entrepreneurship and Socioeconomic Indicators in Latin America. Latin American Research Review, 51(4), 186-201. https://doi.org/10.1353/lar.2016.0055

Amorós, J. E., \& Cristi, O. (2008). Longitudinal analysis of entrepreneurship and competitiveness dynamics in Latin America. International Entrepreneurship and Management Journal, 4(4), 381-399. https://doi.org/10.1007/s11365-008-0082-3

Arango, O. E., Clavijo, S. J., Duque, J., \& Puerta, I. C. (2014). Formación académica, valores, empatía y comportamientos socialmente responsables en estudiantes universitarios. Revista de La Educación Superior, 43(169), 89-105. https://doi.org/https://doi.org/10.1016/j.resu.2015.01.003

Armitage, C., \& Conner, M. (2010). Efficacy of the Theory of Planned Behaviour: A meta- analytic review. British Journal of Social Psychology. https://doi.org/https://doi.org/10.1348/014466601164939

Bandura, A. (2001). Guía para la construcción de escalas de autoeficacia. Revista Evaluar, 2(15), 7-37.

Castro, M. A. B., García, M. L. S., \& Adame, M. E. C. (2015). Hacia una comprensión de los conceptos de emprendedores y empresarios. Suma de Negocios, 6(13), 98-107. https://doi.org/https://doi.org/10.1016/j.sumneg.2015.08.009

Durán, E., \& Arias, D. (2015). Intención emprendedora en estudiantes universitarios: integración de factores cognitivos y socio-personales. Revista Colombiana de Ciencias Sociales, 6(2), 
320. https://doi.org/10.21501/22161201.1528

ESPAE. (2018). GEM 2017. Guayaquil. Retrieved from http://espae.espol.edu.ec/wpcontent/uploads/documentos/GemEcuador2017.pdf

Fitzsimmons, J. R., \& Douglas, E. J. (2011). Interaction between feasibility and desirability in the formation of entrepreneurial intentions. Journal of Business Venturing, 26(4), 431-440. https://doi.org/doi: 10.1016/j.jbusvent.2010.01.001

Galindo, M. Á., \& Méndez, M. T. (2011). La actividad emprendedora y competitividad: factores que inciden sobre los emprendedores. Papeles de Europa, 22(0), 61-75. https://doi.org/10.5209/rev_PADE.2011.v22.3

García, V. D. (2015). Emprendimiento Empresarial Juvenil: Una evaluación con jóvenes estudiantes de universidad. Revista Latinoamericana de Ciencias Sociales, Niñez y Juventud, 13(2), 1221-1236. https://doi.org/DOI:10.11600/1692715x.13246200315

Google Forms. (2018). Cuestionario de Intención Emprendedora CIE. Retrieved from https://docs.google.com/forms/. Accedido junio de 2018

Hair, J. F., Black, W. C., Anderson, R. E., \& Babin, B. J. (2018). Multivariate Data Analysis (8, ilustra ed.). Cengage Learning EMEA.

Henseler, J., Ringle, C. M., \& Sinkovics, R. R. (2009). The use of partial least squares path modeling in international marketing. In New challenges to international marketing. Emerald Group Publishing Limited, 20, 277-319.

Heuer, A., \& Kolvereid, L. (2014). Education in entrepreneurship and the Theory of Planned Behaviour. Accounting, Auditing \&amp; Accountability Journal (Vol. 38). Retrieved from www.emeraldinsight.com

Kautonen, T., van Gelderen, M., \& Tornikoski, E. T. (2013). Predicting entrepreneurial behaviour: A test of the theory of planned behaviour. Applied Economics. https://doi.org/10.1080/00036846.2011.610750

Krueger, N. F., Reilly, M. D., \& Carsrud, A. L. (2000). Competing models of entrepreneurial intentions. Journal of Business Venturing, 15(5-6), 411-432.

Laguía, A., Moriano, J. A., Molero, F., \& Gámez, J. A. (2017). Validación del Cuestionario de Intención Emprendedora en una muestra de estudiantes universitarios de Colombia* Validation of the Entrepreneurial Intention Questionnaire in a Sample of University Students from Colombia. Universitas Psychologica, 16(1), 1-14. 
https://doi.org/10.11144/Javeriana.upsy16-1.vcie

Levy, J.-P., \& Mallou, J. (2006). Modelización con estructuras de covarianzas en ciencias sociales: temas esenciales, avanzados y aportaciones especiales. (C. -Seco, Ed.). España: Netbiblo.

Lortie, J., \& Castogiovanni, G. (2015). The theory of planned behavior in entrepreneurship research: what we know and future directions. International Entrepreneurship and Management Journal, 11(4), 935-957. https://doi.org/https://doi.org/10.1007/s11365-0150358-3

Moreno, J. A. V. (2015). El Emprendimiento Empresarial: La Importancia de ser Emprendedor. IT Campus Academy.

Nunnally, J. C., \& Bernstein, I. H. (1998). Psychometric Theory (McGraw-Hil). New York: McGrawHill.

Robinson, P. B., Stimpson, D. V., Huefner, J. C., \& Hunt, H. K. (1991). An attitude approach to the prediction of entrepreneurship. Entrepreneurship Theory and Practice, 15(4), 13-32. https://doi.org/https://doi.org/10.1177/104225879101500405

Rodriguez, F. (2015). Estudio de la intencion emprendedora el el ámbito científico público. El caso de las ciencias de la vida en España. Universidad de Valencia. Retrieved from https://riunet.upv.es/bitstream/handle/10251/54117/RODRíGUEZ - Estudio de la intención emprendedora en el ámbito científico público. El caso de las ....pdf?sequence=1

Rueda, S., Moriano León, J. A., \& Liñán, F. (2011). VALIDATING A THEORY OF PLANNED BEHAVIOR QUESTIONNAIRE TO MEASURE ENTREPRENEURIAL INTENTIONS. Universidad de Sevilla. Retrieved from https://idus.us.es/xmlui/handle/11441/58964

Salvador, C. M. (2008). Impacto de la inteligencia emocional percibida en la autoeficacia emprendedora. Boletín de Psicología, (92), 65-80.

Sánchez, J. C., Boada, J., Prizmic, A. J., \& Hernández, B. (2014). Pyschometric properties and the factor structure of the Spanish version of the Cognitive Adaptability Scale (MAC). Universitas Psychologica, 13(1), 311-320. https://doi.org/doi:10.11144/ Javeriana.UPSY131.ppfs

Schnarch, A. (2014). Emprendimiento exitoso: cómo mejorar su proceso y gestión. Bogotá: Ecoe Ediciones.

Schumacker, R. E. (2017). Interaction and nonlinear effects in structural equation modeling. New York: Routledge. 
Shapero, A., \& Sokol, L. (1982). The social dimensions of entrepreneurship. Encyclopedia of Entrepreneurship, 72-90.

Soria, K., Zuniga, S., \& Ruiz, S. (2016). Educación e intención emprendedora en estudiantes universitarios: un caso de estudio. Formación Universitaria, 9(1), 25-34. https://doi.org/http://dx.doi.org/10.4067/S0718-50062016000100004

Torres, T., Barreto, I., \& Vásquez, J. C. R. (2015). Creencias y normas subjetivas como predictores de intención de comportamiento proambiental. Suma Psicológica, 22(2), 86-92. Retrieved from http://www.redalyc.org/pdf/1342/134242609003.pdf

Valencia, J. A., Montoya, I., \& Montoya, A. (2014). Factores explicativos de las intenciones emprendedoras en estudiantes universitarios. Revista Espacios, 36(05). Retrieved from http://www.revistaespacios.com/a15v36n05/15360507.html 